\title{
State-of-the-Art in MAC Protocols for Underwater Acoustics Sensor Networks*
}

\author{
Hung T. Nguyen, Soo-Young Shin, and Soo-Hyun Park ${ }^{* *}$ \\ Graduate School of BIT, Kookmin Univ. \\ bluekite315@yahoo.com, \{sy-shin, shpark21\}@kookmin.ac.kr
}

\begin{abstract}
Many potential applications such as ocean sampling network, environment monitoring, undersea exploration, disaster prevention, assisted navigation, and mine reconnaissance can be provided by deploying the Underwater Acoustic Sensor Networks. Because of the peculiarities of acoustic communication in underwater, the MAC protocol which play a role of managing and controlling the channels, must overcome the required of energy consumption, propagation delay and time synchronize as well as other factors. In this paper, we summarize and classify some current proposed MAC protocols as well as make a comparison table in order to bring out the current development of a very interesting research area. Beside, we briefly introduce our suggestion of MAC mechanisms for Underwater Acoustic Sensor Networks (UWASNs) named Gain-time and Guard-time TDMA mechanism and UWANAV mechanism.
\end{abstract}

Keywords: Underwater Acoustic Sensor Networks, Multiple Access Control, Medium Access Control, Schedule-based protocol, Contention-based protocol.

\section{Introduction}

The development of science and technology makes human's desire to conquer the ocean to be more reality by deploying the UWASNs. There many potential applications can be provided throught the networks such as ocean sampling network, environment monitoring, undersea exploration, disaster prevention, assisted navigation, and mine reconnaissance [1]. But the peculiarities of the physicals phenomena in ocean cause some challenges for the scientist when designing the UWASNs like, bandwidth limited, signal attenuation by multipath and fading effects, propagation delay, high bit error rates and temporary loss of connectivity, battery power limited, and underwater sensor failure [2]. These points require suitable architecture for underwater sensor network compare to terrestrial sensor network, especially in MAC protocol.

\footnotetext{
* This research was supported by the MIC (Ministry of Information and Communication), Korea, under the 2007 ITRC (Information Technology Research Center) support program supervised by the IITA (Institute of Information Technology Assessment).

** Corresponding Author.
} 
MAC layer has the objectives of managing and controlling communication channels, which are shared by many nodes to avoid collisions and maintain reliable transmission condition. In terrestrial on air network, $40 \%$ of network utilization depends on MAC layer. But in UWASNs, MAC layer has more important affection on the network utilization. Because of the harsh environment in underwater, MAC protocol for UWASNs must overcome the required of energy consumption, propagation delay and time synchronize as well as other factors. Recently, many new scheduling and synchronization methods have been proposed to solve these problems.

In this paper we summarize the current proposed MAC protocols for UWASNs. The multiple access control including Frequency Division Multiple Access (FDMA), Time Division Multiple Access (TDMA), Code Division Multiple Access (CDMA) and the medium access control including ALOHA, Carrier Sense Multiple Access (CSMA), Multiple Access with Collision Avoidance (MACA), and Floor Acquisition Multiple Access (FAMA), which use in terrestrial sensor network, have been modified in order to adapt with the differences of acoustic transmission in underwater environment. Some typicality of current MAC protocols will be classified and introduced here. We hope that it will be a good reference for who have interesting in wireless sensor network, particularly in UWASNs.

This paper is organized as follows: Section 2 discusses about the multiple access control MAC protocols. Section 3 describes the medium access control MAC protocols. A comparison table in Section 4 will provide a summery in current development of MAC layer for UWASNs. Our proposed MAC mechanism for underwater acoustic sensor networks is presented in section 5. We give our conclusion in Section 6.

\section{Multiple Access Controls}

We make a distinction between multiple access controls schemes such as FDMA, TDMA, and CDMA, can be associated with the physical layer (PHY) and multiple access protocols, situated at the medium access control (MAC) above the PHY [3].

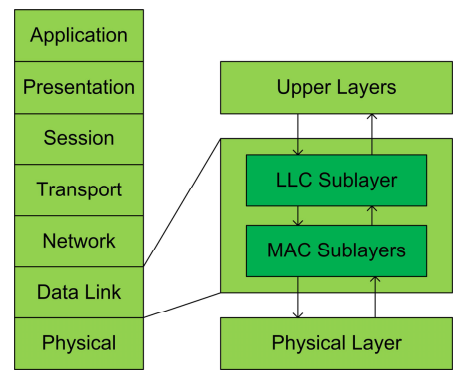

Fig. 1. Open systems interconnection reference model and data link layer architecture 
In OSI layers shows in figure 1, the combination of multiple access controls schemes and medium access controls makes the MAC sub-layer. Roughly speaking, the scheme provide the capability of dividing the total resources available to a base station into individual portions, which can be assigned to different users, and the protocols govern access to the resource portions, e.g. provide access arbitration.

\subsection{FDMA}

In FDMA the system signaling are divided along the communication frequency into non-overlapping channels, and each user is assigned a different frequency channel. The channels often have guard bands between them to compensate for imperfect filters, adjacent channel interference, and spectral spreading due to Doppler. If the channels are sufficiently narrowband then even if the total system bandwidth is large, the individual channels will not experience frequency-selective fading [4].

The severe fading present in underwater acoustic channels creates a difficult environment for FDMA-based systems. And it is an inefficient protocol for the underwater environment because the limited bandwidth which was verified in the Seaweb project 1998 and 1999 [5]. For these reasons, FDMA is not suitable for UWASNs.

\subsection{TDMA}

TDMA is digital transmission technology that divides the communication frequency into time slots and then allocates unique time slots to each user. Users take turn transmitting and receiving in a round-robin fashion. It is worth noting, however, that only one user is actually using the channel at any given time for the duration of a time slot [6]. TDMA also has the advantage that it is simple to assign multiple channels to a single user by simply assigning him multiple time slots.

TDMA has good energy efficiency, but requires strict time synchronization and is not flexible to changes in the number of nodes. Due to the characteristics of the underwater environment it is very challenging to realize a precise synchronization, with a common timing reference, which is required for a proper utilization of time slots in TDMA. Moreover, due to the high delay and delay variance of the UWA channel, TDMA efficiency is limited because of the high guard time required to implement it.

\subsection{CDMA}

In CDMA the information signals of different users are modulated by orthogonal or non-orthogonal spreading codes. The resulting spread signals simultaneously occupy the same time and bandwidth. The receiver uses the spreading code structure to separate out the different users. The most common form of CDMA is multiuser spread spectrum with either direct sequence (DS) or frequency hopping (FH) [4].

CDMA is the most promising physical layer and multiple access technique for UWASNs since when it is robust to frequency-selective fading, compensates for the 
effect of multipath by exploiting Rake filters at the receiver, and allows receivers to distinguish among signals simultaneously transmitted by multiple devices [7].

In the differences projects related to underwater acoustic communication realized by GESMA and its main partners, Sercel Bres ad ENST Bretagne, CDMA technique is carried out in 2003 and 2004 to provide underwater acoustic network with the ability to exchange data in multiple access environment [8]. This technique is a good choice for those who want to realize underwater acoustic communication in a multiuser context or ling range acoustic transmission. According to the deterioration of spreading codes orthogonality brought by multipath, a Rake receiver is also used and evaluated during these sea-trials.

\section{Medium Access Controls}

The main objective of most MAC layer protocols is to reduce energy waste caused by collisions, idle listening, overhearing and excessive overhead. These protocols can be categorized into two main groups: contention based and schedule based MAC protocols. Contention based MAC layer protocol avoids pre-allocation of resources to individual users. Instead, a single communication channel is shared by all users and allocated on-demand. Simultaneous attempts to access the communication medium, however, results in collision. The main objective of contention based MAC layer protocol is to minimize, rather than completely avoid, the occurrence of collisions. To reduce energy consumption, these protocols differ in the mechanism used to reduce likelihood of a collision while minimizing overhearing and control traffic overhead. Schedule based protocol are class of deterministic MAC layer protocol in which access to the channel is based on a schedule. Channel access is limited to one user at a time. This is achieved based on pre-allocation of resources in to individual users [6].

\subsection{Contention-Based MAC Protocols}

\subsubsection{ALOHA Based Protocols}

The original ALOHA protocol is based on random access of users to the medium and do not try to prevent packet collision. Whenever a user has information to send, it transmits it immediately. This naturally leads to a large number of collisions, and hence a number of data packets have to be retransmitted. Therefore, the effective throughput of the ALOHA channel is very low because the probability of packet collisions is high. The Slotted ALOHA scheme was developed to deal with the collision problem. In Slotted ALOHA, the time is divided into slots, and packet transmission is restricted to these time slots. Thus, the number of collisions is reduced significantly. The throughput with Slotted Aloha is double that with basic ALOHA.

The limitation of ALOHA protocol in underwater environment was analyzed in the papers [9] and [10]. In [9], the paper presents a study on ALOHA and Slotted 
ALOHA protocols for UWASNs. The results show that long propagation delay of acoustic signals prohibits the coordinate among nodes so it does not yield any performance gain. Although, the nodes sent the messages in pre-defined time slot, there is no guarantee that they will arrive in time slots. The simple analysis and simulation results show that Slotted ALOHA exhibits the same utilization as nonSlotted ALOHA. More over, in [10], the paper identifies the challenges of modeling contention-based medium access control protocols and presents a model for analyzing ALOHA variants for a simple string topology as a first step toward analyzing the performance of contention-based proposals in multi-hop underwater acoustic sensor networks. The limitation factor in the performance of ALOHA variants is collisions. Avoiding collisions is the goal of refinements to this protocols class.

In order to deploy the ALOHA protocol for UWASNs, adaptive improvements will be added to the original ALOHA overcoming the technical issues of this protocol. In [11] study the performance of ALOHA-based protocols in underwater networks, and propose two enhanced schemes, namely, ALOHA with collision avoidance (ALOHACA), and ALOHA with avoidance notification (ALOHA-AN), which are capable of using the long propagation delays to their advantage. Between two protocols, ALOHA-CA is simpler and more scalable, as it only needs a small amount of memory, and does not rely on additional control messages. ALOHA-AN, on the other hand, requires the use of additional notification (NTF) packets, which serve as advance notification to neighboring nodes, so that they can avoid transmitting packets that could result in collisions. The ALOHA-AN needs to collect and store more information, therefore it requires more resources than ALOHA. Simulation results have shown that both schemes can boost the throughput by reducing the number of collisions, and, for the case of ALOHA-AN, also by reducing the number of unproductive transmissions.

\subsubsection{CSMA Based Protocols}

In CSMA protocol, only one user can transmit at a time, on a first come, first served bases. When a user has a data to transmit it first listens to the medium and senses for a carrier on the medium to determine whether other users are transmitting: the carrier-sense phase. If the medium is busy, the user has to wait until the medium is idle. If no other users transmit data, the user proceeds to transmit its data onto the medium. However, when the medium is busy the user waits for a random of time (back-off time) after a collision before sensing the channel again. If two or more users simultaneously try to transmit, a collision of frames occurs, and all data is corrupted. In this case, all corresponding users stop transmitting. Therefore, after a collision, all the users involved will start contention, each after a randomly chosen amount of time. After finishing the transmission of data, a user waits for an acknowledgement (ACK) from the destination user. If the ACK does not arrive, the user retransmits the data [6].

In [12] the use of acoustic communication in terrestrial wireless sensor networks had been explored as an alternative way of communication among the motes. A lightweight, configurable MAC layer which adopts the principle of CSMA was developed 
in order to facilitate the use of acoustic layer communication. The implementation of Acoustic MAC layer have the capability of snooping on traffic over the acoustic signals feature with a tone detector, automatic ACK after a node receives a package feature. A set of interfaces that allow network services to dynamically configure the Acoustic MAC is also provided. This research have meaningful because it showed some issues of acoustic communication not only in terrestrial wireless sensor network including receiver sampling period, sounder delay and saturation in tone detector but also much more important in UWASNs.

In [13], the multiple-access with collision avoidance and acknowledgment (MACAW) channel access approach for the MAC layer was proposed. The MACAW protocol relies on the exchange of request-to-send (RTS) and clear-to-send (CTS) packets to contend and secure the channel before a data packet is sent followed by an ACK packet. In implementing in such a scheme in ad hoc UWASNs, there is a proposed modification for multiple RTS in contention of the channel.

In [14] the authors also utilize a MAC protocol based on MACA that uses RTS/CTS/DATA/ACK handshaking along with carrier sensing. The protocol similar to MACA and FAMA in multiple communication channels in an Autonomous Underwater Vehicles (AUV) network. A variation of the MACA protocol called MACA-MCP since it utilizes Multiple Channels and Positioning information has been developed with performance enhancements through packet trains and position information exchange. This achieves a self-organizing clustered network behavior that lead to good efficiency and data rates per node in the underwater AUV.

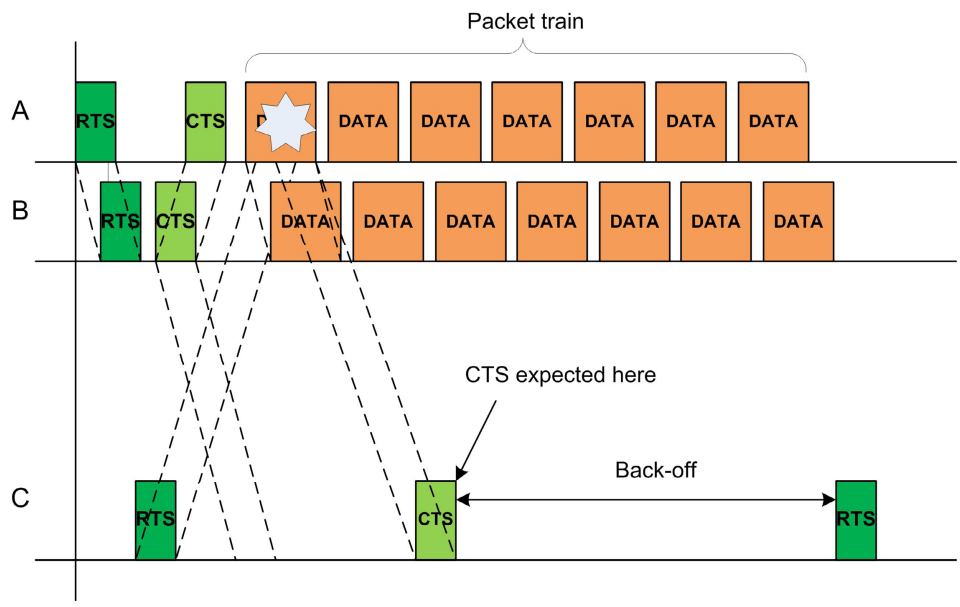

Fig. 2. MACA with DATA packet train improvement

The classical hidden node collision problem of MACA in Figure 2 can be solved by using packet train. The packet train in this case is a single large DATA packet. In such an RTS collision the entire DATA packet is lost and for re-transmission, the complete RTS/CTS/DATA/ACK exchange has to be repeated needlessly wasting 
bandwidth. However, when packet trains are used, the RTS collisions only affect some of the packets in the train and the ACK will indicate this. By using fairly large number of packets in the train, efficiency can greatly be improved.

In [15], the paper proposed a MAC protocol suitable for an underwater acoustic network based on a channel access discipline called FAMA which combines both carrier sensing (CS) and dialogue between the source and receiver prior to data transmission. The new protocol uses time slotting and is thus called Slotted FAMA. Each packet (RTS, CTS, DATA or ACK) has to be transmitted at the beginning of one slot. The slot length has to be determined in a manner that ensures absence of data packet collisions. An ARQ protocol has also been included by sending ACK or NACK (Negative-acknowledment) packets to acknowledge the data reception. Time slotting eliminates the need for excessively long control packets, thus providing savings in energy. Simulation results of this protocols show that for a given network topology, the transmission range can be chosen so as to maximize the network performance in terms of throughput and delay.

\subsection{Schedule-Based MAC Protocols}

In [16], the paper describes a new protocol for a network of acoustically-linked subsea sensors under the Acoustic Communication Network for the Monitoring of the Underwater Environment in Coastal Areas (ACME) project. The main aim of the project was to investigate the development of a system capable of operating in a harsh communication environment such as an estuary. ACME network protocol, ACMENet, is a master-slave protocol intended for small to medium sized UWASNs with arbitrary topologies. And MAC in ACMENet is based on scheduled transmission schedules that are designed such that data packets from slave nodes arrive at the master node consecutively, without collisions. CDMA scheme is used as an asynchronous multi-user transmission scheme where the nodes may be scheduled so that groups of synchronized data packet are received simultaneously. Each packet in the group will use a different orthogonal channel described by the multi-user scheme. Further, the transmission schedules are piggy-backed by special instructions to control the transmission power levels and modulation rates at slave nodes. Once all data packet have been received, the master node will then cause the sequence to be repeated by broadcasting other transmit instruction to the remote sensor nodes.

In [7] UW-MAC, a distributed MAC protocol for underwater acoustic sensor networks, was proposed. It is a transmitter-based CDMA scheme that incorporates a closed-loop distributed algorithm to set the optimal transmit power and code length. It is proven that UW-MAC manages to simultaneously achieve high network throughput, limited channel access delay, and low energy consumption in deep water communications, which are not severely affected by multipath. In shallow water communications, which are heavily affected by multipath, UW-MAC dynamically finds the optimal trade-off among these objectives.

In [17], the paper focus on design an energy-efficient MAC protocol for short range, acoustic sensor networks called "Tone Lohi". Lohi provides an energy conserving, throughput efficient, fair, and stable medium access for acoustic 
networks. The energy is conserved in two ways: first, using data reservations to ensure no data packets collide. Second, employing wake-up tone hard ware that resolves reservation contention with extremely low energy cost. Three flavors of TLohi representing different design choices also propose. By simulation, the results show that ST-Lohi (Synchronized T-lohi) is the most energy efficient protocol, within $3 \%$ of optimal energy. aUT-Lohi (Aggressive Unsynchronized T-Lohi) achieves the highest throughput $(\sim 50 \%$ channel utilization). cUT-Lohi (Conservative Unsynchronized T-Lohi) provides the most robust packet delivery with almost no packet loss. All three flavors exhibit efficient channel utilization, stable throughput, and excellent energy efficiency.

In [18], a distributed, scalable, energy-efficient MAC protocol that works despite long, unknown propagation delays of the underwater acoustic medium. This protocol can be used for delay-tolerant applications such as underwater ecological sensor networks between energy-limited nodes. The protocols differs significantly from ALOHA, MACA, and MACAW protocols in that energy is the main performance metric in the case rather than bandwidth utilization.

The proposed scheme was showed that provides at least $95 \%$ energy-efficiency in the MAC layer, when the number of 1-hop neighbors is about 6. For application, this MAC protocol will be combined with topology control for the operation of energylimited sensor nodes in UWASNs.

\subsection{Hybrid Protocols}

In [19], the paper present a multiple access scheme based on clustering which provides efficiency scalability by spatial reuse of channel resources. The network is partitioned into cluster, and transmissions in each cluster are scheduled following a TDMA algorithm. CDMA is used to enable spatial reused of slots throughout the network. Network scalability is attained by reusing CDMA codes in distant clusters. Connectivity between nodes in different clusters is achieved using receivers that capable of simultaneous detection at multiple spreading codes. The effect of CDMA processing gain on the network performance was quantified through the node connectivity/delay trade-off.

\section{Comparison}

In this section, we compare the protocols which describe in the early part. UW-MAC CDMA is the protocol that leverages CDMA properties to achieve multiple access in the bandwidth-limited underwater channel. Slotted FAMA with handshaking mechanism may lead to low system throughput and sensing an idle channel while in a transmission duration may cause packet collisions. MAC protocol in [18] has Energyefficient operation, but lacks effective mechanism for contention and only suited for applications that have extremely low traffic rates.

Through following comparison table, we bring out a clear picture of the technique solutions using in these protocols in order to satisfy the requirement of collision avoidance, energy consumption and throughput that are very important measurements for the efficiency of a UWASNs. 
Table 1. UWASNs MAC protocols' specifies comparison

\begin{tabular}{|c|c|c|c|c|c|}
\hline $\begin{array}{l}\text { Classifi- } \\
\text { cation }\end{array}$ & Protocols & $\begin{array}{l}\text { Network } \\
\text { topology }\end{array}$ & $\begin{array}{l}\text { Collision } \\
\text { Avoidance }\end{array}$ & $\begin{array}{c}\text { Energy } \\
\text { consumption }\end{array}$ & Throughput \\
\hline \multirow{3}{*}{$\begin{array}{l}\text { Conten- } \\
\text { tion based } \\
\text { protocol }\end{array}$} & \begin{tabular}{|l|} 
[11] ALOHA-CA \\
with header \\
segment and \\
data segment, \\
ALOHA-AN with \\
advance \\
notification \\
packet
\end{tabular} & $\begin{array}{l}\text { - Distributed } \\
\text { topology }\end{array}$ & $\begin{array}{l}\text { - Providing the } \\
\text { local database } \\
\text { table. }\end{array}$ & $\begin{array}{l}\text { Saving by: } \\
\text { - Not transmit } \\
\text { packets may } \\
\text { cause collision }\end{array}$ & \begin{tabular}{|l} 
Boost by \\
reduce: \\
- Collision \\
- Number of \\
unproductive \\
transmissions
\end{tabular} \\
\hline & $\begin{array}{l}\text { [14] MACA with } \\
\text { RTS / CTS/ } \\
\text { DATA / ACK } \\
\text { with carrier } \\
\text { sensing }\end{array}$ & $\begin{array}{l}\text { - Small AUV } \\
\text { network }\end{array}$ & $\begin{array}{l}\text { - Using short } \\
\text { RTS and CTS } \\
\text { - Better tuning } \\
\text { of back-off } \\
\text { timers }\end{array}$ & $\begin{array}{l}\text { - Achieve } \\
\text { efficiency }\end{array}$ & $\begin{array}{l}\text { Increase date } \\
\text { rate due to: } \\
\text { - Dividing the } \\
\text { traffic across } \\
\text { the multiple } \\
\text { channels } \\
\text { - Optimizing } \\
\text { packet train } \\
\text { size }\end{array}$ \\
\hline & \begin{tabular}{|l|} 
[15] Slotted \\
FAMA with \\
carrier sensing \\
(CS) and a \\
nodes' dialogue
\end{tabular} & $\begin{array}{l}\text { - Mobil ad hoc } \\
\text { underwater } \\
\text { network }\end{array}$ & $\begin{array}{l}\text { - RTS or CTS } \\
\text { within } \\
\text { transmission } \\
\text { range over one } \\
\text { slot }\end{array}$ & \begin{tabular}{|l|} 
Saving energy \\
due to: \\
- Eliminating \\
long excessive \\
control packets \\
- Idle state of \\
terminals in \\
time slots
\end{tabular} & $\begin{array}{l}\text { - Achieved } \\
\text { maximum } \\
\text { around } 2 \mathrm{~km} \\
\text { distance }\end{array}$ \\
\hline \multirow{3}{*}{$\begin{array}{l}\text { Schedule } \\
\text { based } \\
\text { protocol }\end{array}$} & $\begin{array}{l}\text { [7] UW-MAC } \\
\text { CDMA using } \\
\text { closed-loop } \\
\text { distributed } \\
\text { algorithm }\end{array}$ & $\begin{array}{l}- \text { Two- } \\
\text { dimensional } \\
\text { deep water } \\
\bullet \text { Three- } \\
\text { dimensional } \\
\text { shallow water }\end{array}$ & $\begin{array}{l}\text { - Small EH } \\
\text { randomly } \\
\text { accessing the } \\
\text { channel }\end{array}$ & \begin{tabular}{|l|} 
Optimal \\
transmit power \\
by: \\
$\bullet$ Have a low \\
number of \\
packet \\
retransmissions
\end{tabular} & $\begin{array}{l}\text { High network } \\
\text { throughput } \\
\text { due to: } \\
\text { • High channel } \\
\text { reuse }\end{array}$ \\
\hline & \begin{tabular}{|l|}
$17]$ Tone Lohi \\
with reservation \\
tone and wake- \\
up tone
\end{tabular} & $\begin{array}{l}\text { - Differences } \\
\text { underwater } \\
\text { sensor network } \\
\text { architecture } \\
\text { scenarios } \\
\end{array}$ & - Reservation & \begin{tabular}{|l|} 
Reduce the \\
energy by: \\
- Using wakeup \\
tone detection
\end{tabular} & $\begin{array}{l}\text { - Efficient at low } \\
\text { load } \\
\text { - Stable at high } \\
\text { load }\end{array}$ \\
\hline & $\begin{array}{l}\text { [18] Distributed, } \\
\text { scalable, } \\
\text { energy-efficient } \\
\text { MAC protocol }\end{array}$ & $\begin{array}{l}- \text { Dense } \\
\text { network of } \\
\text { hundreds of } \\
\text { sensors }\end{array}$ & $\begin{array}{l}\text { - SYNC packet } \\
\text { - Guard time } \\
\text { duration. }\end{array}$ & $\begin{array}{l}\text { Saving nodes } \\
\text { energy due to: } \\
\text { - Sleep mode } \\
\text { - Low duty } \\
\text { cycles }\end{array}$ & $\begin{array}{l}\text { Have low } \\
\text { throughput by: } \\
\text { - Spending time } \\
\text { to wake up } \\
\text { sleep nodes }\end{array}$ \\
\hline
\end{tabular}




\section{Suggestion Mechanism of the UWASNs MAC}

\subsection{GT $^{2}$ TDMA MAC}

Common problem of underwater environment is a propagation delay regardless of what kinds of MAC are used. That is, the problem of propagation delay is not related to communication protocols. Generally, a Guard-time has been applied based on the maximum propagation delay of the network. However, our Gain-time and Guard-time $\left(\mathrm{GT}^{2}\right)$ TDMA MAC scheduling proposed technique can be applicable to clustered networks. The proposed method is to increase the network efficiency by determining the moment of data Gain-time and Guard-time in to consideration [23].

\subsection{UWA-NAV: Energy Efficient Error Control Scheme for Underwater Acoustic Sensor Network}

Since there are various disturbing factors such as long propagation delay and high error rate in UW-ASN, an error control mechanism is required to improve the system performance efficiently. The carrier sensing technique, which detects whether the public media access is already occupied or not and evade the collision, is used as an error control mechanism. A method of monitoring the energy level of wireless frequency in physical layers and a method of setting-up NAV (Network Allocation Vector) using the frame transmission period were modified and re-defined for underwater environment. Through these works, a more efficient UWA-NAV technique was able to be proposed and the performance of the proposed technique was evaluated and compared with the case without UWA-NAV [24].

\section{Conclusion}

This paper reviews the current development of MAC protocols for Underwater Acoustic Sensor Networks. These protocols were introduced and classified by legacy MAC scheme likes contention-based protocols and schedule-based protocols. A table also was added in order to bring a comparison between specifies of the represent UWASNs MAC. We also implement our proposed GT $^{2}$ TDMA and UWA-NAV MAC mechanisms.

Our researches are still going on the Underwater Acoustic Sensor Networks project supported by the MIC (Ministry of Information and Communication), Korea, under the ITRC (Information Technology Research Center) support program supervised by the IITA (Institute of Information Technology Assessment). Our future work including simulation our proposed MAC mechanisms (GT ${ }^{2}$ and UWA-NAV), experiment in the filed test in the sea with fully implementation by using MSP430 microcontroller, acoustic modem as well as others hardware and software. We also will execute the comparison between simulation and experiment in order to take the implementation for our mechanisms. 


\section{References}

[1] Akyildiz, I.F., Pompili, D., Melodia, T.: Underwater acoustic sensor networks: research challenges, Ad Hoc Networks, vol. 3(3), pp. 257-279. Elsevier, Amsterdam (2005)

[2] Akyildiz, I.F., Pompili, D.: State-of-the-art in protocol research for underwater acoustic sensor networks. In: WUWNet 2006. Proceedings of the 1st ACM international workshop on Underwater networks (2006)

[3] Brand, A., Aghvami, H.: Multiple Access Protocols for Mobile Communications: GPRS, UMTS and Beyond. Wiley Publication (2002)

[4] Wu, S.L., Tseng, Y.C.: Wireless Ad Hoc Networking: Personal-Area, Local-Area, and the Sensory-Area Networks, 1st edn. Auerbach Publications (March 2007)

[5] Rice, J., et al.: Evolution of Seaweb Underwater Acoustic Networking. In: Oceans Conf., Providence, RI, pp. 2007-2017 (2000)

[6] Sohraby, K., Minoli, D., Znati, T.: Wireless Sensor networks: Technology, Protocols, and Applications. Wiley Publication, Chichester (2007)

[7] Pompili, D., Melodia, T., Akyildiz, I.F.: A Distributed CDMA Medium Access Control for Underwater Acoustic Sensor Networks. In: Proc. of Mediterranean Ad Hoc Networking Workshop (Med-Hoc-Net), Corfu, Greece (2007)

[8] Lapierre, G., Beuzelin, N., Labat, J., Trubuil, J., Goalic, A., Saoudi, S., Ayela, G., Coince, P., Coatelan, S.: 1995-2005: Ten years of active research on underwater acoustic communications in Brest. In: Oceans 2005 - Europe, vol. 1, pp. 425-430 (June 20-23, 2005)

[9] Vieira, L.F.M., Kong, J., Lee, U., Gerla, M.: Analysis of ALOHA protocols for underwater acoustic sensor networks. In: WUWNet. The First ACM International Workshop on UnderWater Networks (2006)

[10] Gibson, J.H., Xie, G.G., Xiao, Y., Chen, H.: Analyzing the Performance of Multi-hop Underwater Acoustic Sensor Networks. In: Proc. MTS/IEEE Oceans 2007 Conference, Scotland (June 2007)

[11] Chirdchoo, N., Soh, W.S., Chua, K.C.: ALOHA-based MAC Protocols with Collision Avoidance for Underwater Acoustic Networks. In: INFOCOM 2007, 26th IEEE International Conference on Computer Communications, IEEE, Los Alamitos (2007)

[12] Zhang, J., Huang, Z., Liu, X.: Acoustic Communication in Wireless Sensor Networks. Department of Computer Science University of Virginia, CS651, Wireless Sensor Networks (TA Course) (December 2005)

[13] Foo, K.Y., Atkins, P.R., Collins, T., Morley, C., Davies, J.: A routing and ChannelAccess Approach for an Ad Hoc Underwater Acoustic Network. In: OCEANS 2004. MTS/IEEE TECHNO-OCEAN 2004, vol. 2, pp. 789-795 (November 9-12, 2004)

[14] Shahabudeen, S., Chitre, M., Motahi, M.: A multi-channel MAC protocol for AUV networks. In: Oceans 2007, Aberdeen (June 2007)

[15] Molins, M., Stojanovic, M.: Slotted FAMA: a MAC Protocol for Underwater Acoustic Networks. In: OCEANS. Proc. of MTS/IEEE Conference and Exhibition for Ocean Engineering, Science and Technology, Boston, MA (September 2006)

[16] Adams, A.E., Acar, G.: An Acoustic Network Protocol for Sub-Sea Sensor Systems. In: IEEE Oceans 2005 Europe Conferene, Brest (2005)

[17] Syed, A.A., Ye, W., Heidemann, J.: T-Lohi: A New Class of MAC Protocols for Underwater Acoustic Sensor Networks, in Technical Report ISI-TR-638, USC/Information Sciences Institute (April 2007) 
[18] Rodoplu, V., Park, M.K.: An Energy-Efficient MAC Protocol for Underwater Wireless Acoustic Networks. In: OCEANS. Proc. Of MTS/IEEE Conference and Exhibition for Ocean Engineering, Science and Technology (September 2005)

[19] Salva-Garau, F., Stojanovic, M.: Multi-cluster Protocol for Ad Hoc Mobile Underwater Acoustic Networks. In: Proc. of MTS/IEEE OCEANS, San Francisco, CA (September 2003)

[20] Sozer, E., Stojanovic, M., Proakis, J.: Underwater Acoustic Networks. IEEE Journal of Oceanic Engineering 25(1), 72-83 (2000)

[21] Heidemann, J., Ye, W., Wills, J., Syed, A., Li, Y.: Research challenges and applications for underwater sensor networking. In: WCNC 2006. Wireless Communications and Networking Conference, vol. 1, pp. 228-235. IEEE, Los Alamitos (2006)

[22] Partan, J., Kurose, J., Levine, B.N.: A survey of practical issues in underwater networks. In: WUWNet 2006. Proceedings of the 1st ACM international workshop on Underwater networks, pp. 17-24. ACM Press, New York (2006)

[23] Shin, S.Y., Park, S.H.: GT2: Reduced Wastes time Mechanism for Underwater Acoustic Sensor Network. In: Denko, M., et al. (eds.) EUC Workshops 2007. LNCS, vol. 4809, Springer, Heidelberg (2007)

[24] Shin, S.Y., Park, S.H.: UWA-NAV: Energy Efficient Error Control scheme for Underwater Acoustic Sensor Network. In: Denko, M., et al. (eds.) EUC Workshops 2007. LNCS, vol. 4809, Springer, Heidelberg (2007) 